\title{
Clinical features, treatment and risk factors for interstitial pneumonia in B-cell non-Hodgkin lymphoma patients
}

\author{
Cong $\mathrm{Li}^{1,2,3}$, Fangxiao $\mathrm{Lu}^{4}$, Tao Lei ${ }^{1,2,3}$, Haifeng Yu ${ }^{1,2,3}$, Haiyan Yang ${ }^{1,2,3}$ \\ ${ }^{1}$ Department of Medical Oncology, Institute of Cancer and Basic Medicine (ICBM), Chinese Academy of Sciences, Hangzhou, China; ${ }^{2}$ Department \\ of Medical Oncology, Cancer Hospital of the University of Chinese Academy of Sciences, Hangzhou, China; ${ }^{3}$ Department of Medical Oncology, \\ Zhejiang Cancer Hospital, Hangzhou, China; ${ }^{4}$ Department of Medical Imaging, Zhejiang Cancer Hospital, Hangzhou, China \\ Contributions: (I) Conception and design: H Yang, C Li; (II) Administrative support: H Yang; (III) Provision of study materials or patients: H Yu, T \\ Lei; (IV) Collection and assembly of data: C Li, F Lu; (V) Data analysis and interpretation: C Li; (VI) Manuscript writing: All authors; (VII) Final \\ approval of manuscript: All authors. \\ Correspondence to: Haiyan Yang. Department of Medical Oncology, Institute of Cancer and Basic Medicine (ICBM), Chinese Academy of Sciences; \\ Cancer Hospital of the University of Chinese Academy of Sciences; Zhejiang Cancer Hospital, No. 1 East Banshan Road, Hangzhou, China. \\ Email: yanghy@zjcc.org.cn.
}

Background: Interstitial pneumonia (IP) is a common and fatal adverse effect of rituximab-containing immunochemotherapy in lymphoma patients. Following prophylactic treatment with trimethoprimsulfamethoxazole (TMP-SMX), the clinical features, treatment, and risk factors for IP development remain largely undefined.

Methods: From April 2015 and April 2018, 294 patients diagnosed with CD20+ B-cell non-Hodgkin lymphoma (NHL) were included in this study. All patients received front-line RCHOP-like chemotherapy and prophylactic treatment of TMP-SMX once daily. We summarized the clinicopathologic characteristics and treatment outcomes of IP in these patients and explored the possible risk factors of IP.

Results: The overall incidence of IP was $8.16 \%$. Typical clinical symptoms included fever for $1-3$ days in 11 patients, dyspnea in 4 patients, expectoration in 5 patients, and dry cough in 7 patients. A total of 8 patients showed no apparent symptoms. Prior to IP, the median number of chemotherapy cycles was 4 . The median time for IP initiation was 63 days, and the median duration of IP treatment was 11 days. All patients recovered from IP after treatment. A total of 6 patients continued to receive chemotherapy without rituximab, and 14 patients received rituximab combined with chemotherapy. No patients experienced IP recurrence. In univariate and multivariate analysis, male, diabetes, low lymphocyte counts $\left(<1.0 \times 10^{9} / \mathrm{L}\right)$ and low CD4/CD8 counts were identified as risk factors of IP. Patients with no risk factors were included in the low-risk group; 1 to 2 factors: intermediate-risk; $\geq 3$ : high-risk. The occurrence of IP differed across three groups (low risk, 0\%; intermediate risk, 7\%; high risk, 14.5\%; $\mathrm{P}=0.059$ ).

Conclusions: The incidence of IP was $8.16 \%$ in patients with CD20+ B-cell NHL. Males, diabetes, low absolute lymphocyte counts (ALC) $\left(<1.0 \times 10^{9} / \mathrm{L}\right)$ and low CD4/CD8 were identified as risk factors of IP.

Keywords: B-cell lymphoma; chemotherapy; interstitial pneumonia (IP); risk factor; rituximab

Submitted Feb 12, 2020. Accepted for publication Aug 05, 2020.

doi: $10.21037 /$ tcr-20-988

View this article at: http://dx.doi.org/10.21037/tcr-20-988 


\section{Introduction}

Chemotherapy containing rituximab regimens are the current front-line therapy for B-cell non-Hodgkin lymphoma (NHL) $(1,2)$, the safety of which have been intensely investigated (3). Several studies have reported that approximately $1.3 \%$ to $14.8 \%$ of patients with NHL developed interstitial pneumonia (IP) during immunochemotherapy $(4,5)$. IP is a heterogeneous group diseases that are classified together because of similar clinical, radiographic, physiologic, or pathologic manifestations. IP can be idiopathic or secondary to different diseases. Among multiple causes of IP, various infectious processes are especially common in immunocompromised hosts. These patients often develop mixed infections with several pathogens, such as pneumocystis jirovecii (PCP), bacterial pneumonias, and viral pneumonias (6). In our institute, patients with B-cell NHL routinely received prophylaxis of trimethoprimsulfamethoxazole (TMP-SMX) during immunochemotherapy, because TMP-SMX is the first choice for prophylaxis and treatment of PCP infections $(6,7)$. However, there were still some patients develop IP. Herein, we summarized the clinicopathologic characteristics and treatment outcomes of IP in these patients and explore possible risk factors of IP. We present the following article in accordance with the STROBE reporting checklist (available at http://dx.doi.org/10.21037/tcr20-988).

\section{Methods}

\section{Patients}

From April 2015, patients diagnosed with B-cell NHL in our hospital were enrolled. WHO criteria were used to confirm histological diagnoses. Immunostaining was used to confirm CD20+ B-cell lymphoma. Patients were administered tri-weekly cyclophosphamide, vincristine, and prednisone (COP), cyclophosphamide, doxorubicin, vincristine, prednisone, and etoposide (EPOCH), or cyclophosphamide, doxorubicin, vincristine, and prednisone (CHOP) regimens combined with rituximab. Patients with acquired immunodeficiency were excluded. Patients with exposure history to occupational and environmental agents, particularly inorganic or organic dusts, and drug-induced pulmonary toxicity were excluded. Patients with rheumatic disease, sarcoidosis, or a history of thoracic radiation were also excluded. All patients received oral prophylactic TMPSMX once per day during the chemotherapy period. Each TMP-SMX regime included an $80 \mathrm{mg}$ dose of TMP and a $400 \mathrm{mg}$ dose of SMX. Baseline characteristics of the patients included patient demographics, performance status, lymphoma subtype, disease stage, medical history and laboratory tests were collected during diagnosis. Ann Arbor classifications were used for disease staging. The study was conducted in accordance with the Declaration of Helsinki (as revised in 2013). The study was approved by ethics board of Zhejiang Cancer hospital (the registration number of ethics board: IRB-2020-19) and informed consent was taken from all the patients.

\section{Diagnosis of IP}

Patients were evaluated by computed tomography (CT) or positron emission tomography-computed tomography (PET/CT) every two cycles of treatment. CT assessments were performed every 3 months upon the completion of chemotherapy. Extra pulmonary CT was performed in patients who developed suspicious pulmonary infection symptoms. The observation period of IP was from the first day of chemotherapy to 9 months following the final chemotherapy dose (8). IP was diagnosed based on radiologic findings, clinical symptoms, laboratory tests, and pathologic results. The necessary radiological findings of IP were diffuse pulmonary interstitial infiltrates, and other imaging manifestation including traction bronchiectasis, bilateral reticular opacities, a loss of lobe volume, and opacity in the lower lungs (9-11). To reduce subjective bias, two independent radiologists reviewed the pulmonary CT images to confirm the diagnosis of IP. For patients diagnosed with IP, CT scans were repeated on a weekly basis until all interstitial infiltrates disappeared. Serum lactate dehydrogenase (LDH), C-reactive protein (CRP) levels, procalcitonin (PCT), lymphocyte cells, serum b-Dglucan, the number of peripheral white blood cells (WBCs) and other serum inflammatory biomarkers were assessed. We collected blood and sputum samples for microbiological assessments. Complete lung function tests and arterial blood gas analysis were performed to assess the severity and pattern of lung involvement. Patients were recommended to receive a bronchoalveolar lavage (BAL) at the time of IP occurrence. Fluids from BAL were sent for cell counts, mycobacterial cultures, viral, and fungal pathogens. In patients with atypical or progressive symptoms, atypical radiographic features, or a poor response to treatment, lung biopsy was considered. The detailed treatment for IP and its outcomes were regularly reviewed throughout the study. 


\section{Statistical analysis}

The chi-square test was used to compare categorical variables between non-IP and IP patients. Univariate and multivariate analyses were compared using logistic regression for the assessment of the risk factors of IP. P values $<0.05$ following univariate analyses were included in the multivariate analyses. Kaplan-Meier (KM) survival curves were plotted. P values $<0.05$ were deemed significant in two-sided tests. Data were analyzed using SPSS version 22.

\section{Results}

\section{Patient characteristics}

Between April 2015 and April 2018, 294 patients who met the inclusion criteria were enrolled. The median aged was 56 years (range, 15 to 82 years). Chemotherapy regimens included RCHOP ( $\mathrm{n}=255,82 \%)$, REPOCH ( $\mathrm{n}=47,15.1 \%)$ and $\operatorname{RCOP}(n=9,2.9 \%)$. The characteristics of all patients are shown in Table 1.

\section{Incidence of IP and treatment outcomes}

All patients received CT prior to treatment, in which no cases of IP manifestations were observed. Following 1-6 cycles of rituximab chemotherapy (median 4 cycles), IP occurred in 24 patients (8.16\%) including 18 males and 6 females. The median age was 58 years (range, 18 to 76 years). Typical clinical symptoms included fever for 1-3 days in 11 patients, dyspnea in 4 patients, expectoration in 5 patients and dry cough in 7 patients. A further 8 patients showed no apparent symptoms. The median time from the initiation of therapy to IP was 63 days (range, 15 to 158 days). At the onset of IP, 3 patients had grade IIIIV neutropenia and received G-CSF treatment. Sputum cultures were assessed in 5 patients with expectoration, and pseudomonas aeruginosa and klebsiella pneumoniae were detected in two patients. Blood cultures were assessed in 6 patients with fever $\left(\geq 38.5^{\circ} \mathrm{C}\right)$ but showed no evidence of pathogens. Six patients were also tested for CMV antigenemia and the results were negative. Bronchoscopy for BAL was performed in 4 patients, but no PCP was detected in the fluid. Other common suspicious pathogens included haemophilus, stenotrophomonas, tropheryma, lexophile, and human gammaherpesvirus 4. A total of 4 patients received arterial blood gas analysis and pulmonary function tests, of which 3 had type I respiratory failure and
2 had mild restrictive defects with reductions in total lung capacity or residual volume.

Immunochemotherapy was withheld after an IP diagnosis, at which time patients were treated empirically with antibiotics or antifungal agents, with the medication adjusted according to aetiology results. Glucocorticoids, including methylprednisolone or dexamethasone were used to inhibit telangiectasia and reduce oedema and exudates. A total of 4 patients experienced fever, cough, absolute lymphocyte counts (ALCs) decreases, and imaging manifestations that suggested virus infection. These patients received ganciclovir treatment and recovered from IP eventually. Chest CT features of 16 patients were diffuse, bilateral, interstitial infiltrates. These patients also showed elevated serum betaD-glucan assays. Although no etiological evidence was obtained, these patients were suspected of PCP infection. Finally, they received therapeutic doses of TMP-SMX.

The median duration of IP treatment was 9 days ( 7 to 58 days). All patients recovered and no patients died of respiratory failure or severe pulmonary infection. A total of 5 patients did not receive immunochemotherapy, whilst 5 patients received subsequent chemotherapy without rituximab. A total of 14 patients received rituximab combined with chemotherapy. No patients developed recurrent IP following retreatment.

\section{Risk factors for IP}

We investigated both clinical and laboratory risk factors through binary logistic regression models (Table 2). Following univariate analysis, males [odds ratio (OR) 3.43, 95\% CI: $1.32-8.9, \mathrm{P}=0.001$ ], diabetic patients (OR 3.48, 95\% CI: $1.17-10.34, \mathrm{P}=0.025)$, lymphocyte counts $\leq 1 \times 10^{9} / \mathrm{L}$ (OR $0.37,95 \%$ CI: $0.16-0.87, \mathrm{P}=0.023$ ) and low CD4/CD8 cells (OR 0.73, 95\% CI: 0.58-0.92, P=0.007) were identified as IP risk factors. Following multivariate analysis, all factors remained significantly associated with IP development.

We stratified patients according to risk factors. Those with no risk factors were included in the low-risk group; 1 to 2 factors: intermediate-risk; $\geq 3$ : high-risk. The occurrence of IP differed across the groups (low risk, $0 \%$; intermediate risk, 7\%; high risk, 14.5\%; $\mathrm{P}=0.059$ ) (Figure 1).

\section{Discussion}

IP is characterized by diffuse parenchymal lung 
Table 1 Baseline characteristics of patients with B-cell non-Hodgkin lymphoma ( $\mathrm{n}=294)$

\begin{tabular}{|c|c|c|c|c|}
\hline Factor & All patients & With IP & Without IP & $p$ value \\
\hline Male sex, $\mathrm{n}(\%)$ & $144(49.0)$ & $18(75.0)$ & $126(46.7)$ & 0.007 \\
\hline Diabetes history, n (\%) & $24(8.2)$ & $5(20.8)$ & $19(7.0)$ & 0.035 \\
\hline Hypertension, n (\%) & $73(24.8)$ & 8 (33.3) & $65(24.1)$ & 0.219 \\
\hline Lung involvement by lymphoma, n (\%) & $14(4.8)$ & $1(4.2)$ & $13(4.8)$ & 0.681 \\
\hline ECOG PS $\geq 2, n(\%)$ & $38(13.3)$ & $2(8.3)$ & $36(13.3)$ & 0.375 \\
\hline B symptoms, n (\%) & $66(22.4)$ & $5(20.8)$ & $61(22.6)$ & 0.539 \\
\hline \multicolumn{5}{|l|}{ Histology, n (\%) } \\
\hline MCL & $3(1)$ & 0 & $3(1.1)$ & \multirow[t]{3}{*}{0.383} \\
\hline SLL/CLL & $4(1.4)$ & 0 & $4(1.5)$ & \\
\hline MALT & $20(6.8)$ & $3(12.5)$ & $17(6.3)$ & \\
\hline \multicolumn{5}{|l|}{ Ann Arbor stage, n (\%) } \\
\hline I & $42(14.3)$ & $4(16.7)$ & $38(14.1)$ & \\
\hline II & 95 (32.3) & $8(33.3)$ & 87 (32.2) & \multirow{3}{*}{0.978} \\
\hline III & $56(19)$ & $4(16.7)$ & $52(19.3)$ & \\
\hline IV & $101(34.4)$ & 8 (33.3) & $93(34.4)$ & \\
\hline LDH (U/L) & $242(102-2,078)$ & 273 (201.5-542) & $238.5(197-328)$ & 0.613 \\
\hline CRP (mg/L) & $4.4(0-193.8)$ & $10.4(8.6-24.1)$ & $4.0(1.2-21.2)$ & 0.155 \\
\hline ESR $(m m / h)$ & $21(1-92)$ & $27(21-36)$ & $21(11-36)$ & 0.42 \\
\hline$\beta_{2}$ microglobulin ( $\left.\mu \mathrm{g} / \mathrm{L}\right)$ & 2111 (189-8213) & 2407 (1821-3337.5) & 2109.5 (1819.8-3043.5) & 0.66 \\
\hline Alb (g/L) & $43.2(22.1-52.5)$ & 42.7 (38.5-45.5) & $43.3(39.8-46.8)$ & 0.689 \\
\hline CD3 & $74.3(8.5-95)$ & 74.5 (64.3-82.2) & 70.1 (66.5-81.9) & 0.496 \\
\hline CD4 & $644.1 \pm 388.0$ & $788.1 \pm 301.0$ & $631.3 \pm 392.7$ & 0.058 \\
\hline CD8 & $24.4(17.3-31.4)$ & 24.7 (17.9-31.8) & $16.9(12.3-32.7)$ & 0.092 \\
\hline CD4/CD8 & $1.6(0.4-7.8)$ & $1.6(1.1-2.4)$ & $3(1.4-4.2)$ & 0.035 \\
\hline
\end{tabular}

$\mathrm{P}$ value refers to the difference between two groups (patients with IP or without IP). *, basic lung disease included chronic obstructive pulmonary disease, pulmonary emphysema, tuberculosis, benign nodules and fibrosis. ECOG, eastern cooperative oncology group; DLBCL, diffuse large B-cell lymphoma; FL, follicular lymphoma; MCL, mantle cell lymphoma; MALT, mucosa-associated lymphoid tissue; CLL, chronic lymphocytic leukemia; SLL, small lymphocytic lymphoma; PS, performance status; BM, bone marrow; IPI, international prognostic index; ALC, absolute lymphocyte count; ESR, erythrocyte sedimentation rate; Alb, albumin. IP, interstitial pneumonia. 
Table 2 Binary logistic regression analysis of risk factors for interstitial pneumonia (IP)

\begin{tabular}{|c|c|c|c|c|c|c|}
\hline Factor & \multicolumn{3}{|c|}{ Univariate analysis } & \multicolumn{3}{|c|}{ Multivariate analysis } \\
\hline Age (>60 vs. $\leq 60)$ & 1.04 & $0.44-2.46$ & 0.94 & & & \\
\hline Diabetes (yes vs. no) & 3.48 & $1.17-10.34$ & 0.025 & 5.69 & $1.66-19.43$ & 0.006 \\
\hline Basic lung disease (yes vs. no) & 1.86 & $0.77-4.49$ & 0.17 & & & \\
\hline Lymphocyte (>1 vs. $\leq 1)\left(10^{9} / \mathrm{L}\right)$ & 0.37 & $0.16-0.87$ & 0.023 & 0.33 & $0.13-0.84$ & 0.02 \\
\hline CD4/CD8 & 0.73 & $0.58-0.92$ & 0.007 & 0.68 & $0.53-0.86$ & 0.002 \\
\hline
\end{tabular}

OR, odds ratio.

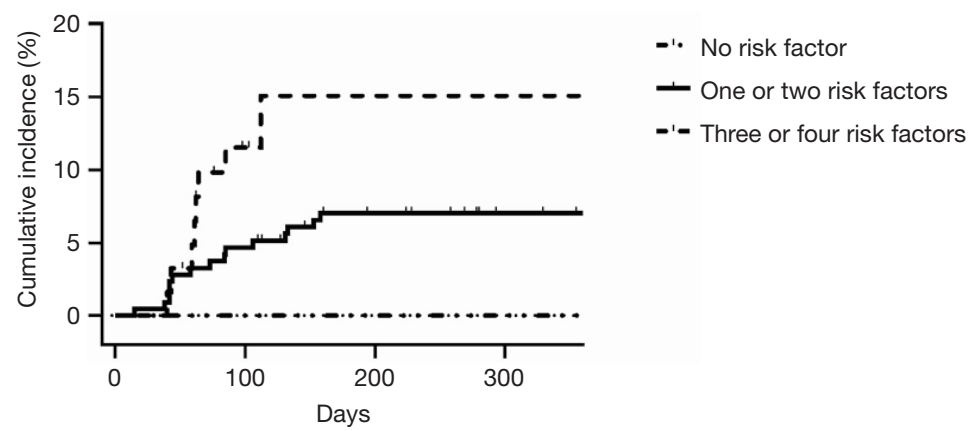

Figure 1 The cumulative incidence of IP according to the number of risk factors (low risk, $0 \%$; intermediate risk, $7 \%$; high risk, $14.5 \%$; $\mathrm{P}=0.059)$. IP, interstitial pneumonia.

filtrates (12) that lead to irreversible vascular and alveolar damage, altered gas exchange, fibrosis, and a loss of pulmonary and cardiopulmonary function (13). Patients diagnosed with IP would experience various pulmonary symptoms and would typically cease chemotherapy, which can influence the efficacy of chemotherapy and shorten patient survival. In this study, patients were in a state of impaired immune function as they have received high-dose glucocorticoid, rituximab and chemotherapy treatment. Pulmonary infections were the most common complication in immunocompromised patients. All patients with IP in this study recovered after antimicrobial treatment, which led us to speculate that the most probable cause of IP was pulmonary infection.

Amongst the possible risk factors for IP, the immune status of patients was critical. For adults, CD4 cell counts of 350 to $499 / \mathrm{mm}^{3}$ and 200 to $349 / \mathrm{mm}^{3}$ indicated mild and severe immunosuppression, respectively. However, several factors such as the season, temperature, drugs and glucocorticoids affected the CD4 cell counts (14). Therefore, the ratio of CD4 and CD8 can be used as a predictor of immune function $(15,16)$. HIV-infected adults should receive primary/secondary PCP prophylaxis for a CD4 counts $<200$ cells $/ \mathrm{mm}^{3}$ (17). In previous studies, CD4 lymphocyte counts $\leq 200 / \mathrm{mm}^{3}$ prior to chemotherapy were indicative of an increased PCP incidence compared to patients with counts $\geq 200 / \mathrm{mm}^{3}(11.8 \%$ vs. $1.5 \%$, $\mathrm{P}=0.045$ ) (18). Wang and colleagues also identified lower levels of CD4 and CD4/CD8 in NHL patients with IP after rituximab therapy, compared to IP negative patients. This trend was further demonstrated through the administration of RCHOP regimens (4).

As B cells dictate CD4 $\mathrm{T}$ cell activation, changes in the ALCs are representative of host immune status. Huang 
and colleagues showed that those with low ALCs presented a 2.75-fold higher risk of IP (19). Katsuya and colleagues also demonstrated that lymphocyte counts $<1,000 / \mathrm{mm}^{3}$ prior to R-CHOP therapy increased the likelihood of PCP development (10). Consistent with these findings, reduced ALC levels $\left(<1.0 \times 10^{9} / \mathrm{L}\right)$ and $\mathrm{CD} 4 / \mathrm{CD} 8$ at diagnosis were predictive of a higher incidence of IP in this study.

A history of diabetes was a further risk factor of IP. An observational study showed that diabetes was associated with a 3-fold higher risk of death after mild-to-moderate community-acquired pneumonia (CAP) (20). In addition, persistent postprandial hyperglycemia was independently associated with late mortality after pneumonia (21). Preclinical studies have linked hyperglycemia to endothelial dysfunction, a loss of chemotaxis, lower granulocyte adherence, reduced phagocytosis, and a loss of microbicidal capacity (22-24). These effects can be in-part reversed by glucose lowering therapies $(25,26)$. In animal models of diabetes, hyperglycaemia was associated with decreased bacterial clearance and higher rates of infection-related mortality (27). Patients with a history of diabetes were more prone to infection during immunochemotherapy.

Although a history of smoking and pre-treatment pulmonary disease were not significant risk factors of IP, these diseases were more prevalent in males than females (73/144 vs. 1/150, 84/144 vs. 72/150, respectively). Although prophylactic treatment of TMP-SMX is available for all patients, the incidence of IP has remained as $8 \%$. For patients with 3 or 4 risk factors, the incidence of IP was as high as $14.5 \%$. Oncologists should therefore pay attention to patients with these risk factors and explore more intensive and effective prophylactic treatments to reduce the incidence of IP.

The pathogenesis of IP was not fully defined in this retrospective study, which was a limitation. Although all patients diagnosed with IP recovered after treatment, we did not obtain pathogenic evidence from each individual patient. Only 4 patients received BAL tests, and it was difficult to interpret the results of BAL fluid analysis. Therefore, further diagnostic measures and etiological exploration are required in future studies.

\section{Conclusions}

This retrospective study showed that 24/294 patients $(8.16 \%)$ with B cell lymphoma treated with first-line RCHOP-like regimens developed IP. Males, diabetes, low absolute lymphocyte counts and low CD4/CD8 numbers were identified as risk factors for IP induction.

\section{Acknowledgments}

We thank Dr Wang Xuefen and Dr Zhang Yigong for discussions regarding IP treatment and statistics analysis.

Funding: The study is supported by Medical Health Science and Technology Project of Zhejiang Provincial Health Commission (2015KYB067).

\section{Footnote}

Reporting Checklist: The authors have completed the STROBE reporting checklist. Available at http://dx.doi. org/10.21037/tcr-20-988

Data Sharing Statement: Available at http://dx.doi. org/10.21037/tcr-20-988

Peer Review File: Available at http://dx.doi.org/10.21037/ tcr-20-988

Conflicts of Interest: All authors have completed the ICMJE uniform disclosure form (available at http://dx.doi. org/10.21037/tcr-20-988). The authors have no conflicts of interest to declare.

Ethical Statement: The authors are accountable for all aspects of the work in ensuring that questions related to the accuracy or integrity of any part of the work are appropriately investigated and resolved. The study was conducted in accordance with the Declaration of Helsinki (as revised in 2013). The study was approved by ethics board of Zhejiang Cancer hospital (the registration number of ethics board: IRB-2020-19) and informed consent was taken from all the patients.

Open Access Statement: This is an Open Access article distributed in accordance with the Creative Commons Attribution-NonCommercial-NoDerivs 4.0 International License (CC BY-NC-ND 4.0), which permits the noncommercial replication and distribution of the article with the strict proviso that no changes or edits are made and the original work is properly cited (including links to both the formal publication through the relevant DOI and the license). See: https://creativecommons.org/licenses/by-nc-nd/4.0/. 


\section{References}

1. Coiffier B, Lepage E, Briere J, et al. CHOP chemotherapy plus rituximab compared with CHOP alone in elderly patients with diffuse large-B-cell lymphoma. N Engl J Med 2002;346:235-42.

2. Pfreundschuh M, Trümper L, Osterborg A, et al. CHOP-like chemotherapy plus rituximab versus CHOPlike chemotherapy alone in young patients with goodprognosis diffuse large-B-cell lymphoma: a randomised controlled trial by the MabThera International Trial (MInT) Group. Lancet Oncol 2006;7:379-91.

3. Winkler U, Jensen M, Manzke O, et al. Cytokine-release syndrome in patients with B-cell chronic lymphocytic leukemia and high lymphocyte counts after treatment with an anti-CD20 monoclonal antibody (rituximab, IDECC2B8). Blood 1999;94:2217-24.

4. Wang Q, Zhang YF, Jia RF, et al. The risk factors and clinical features of interstitial pneumonia in B-cell non-Hodgkin's lymphoma patients who were treated with rituximab-CHOP regimen. China Oncology 2014;24:936-43.

5. Salmasi G, Li M, Sivabalasundaram V, et al. Incidence of pneumonitis in patients with non-Hodgkin lymphoma receiving chemoimmunotherapy with rituximab. Leuk Lymphoma 2015;56:1659-64.

6. Kamel S, O'Connor S, Lee N, et al. High incidence of Pneumocystis jirovecii pneumonia in patients receiving biweekly rituximab and cyclophosphamide, adriamycin, vincristine, and prednisone. Leuk Lymphoma 2010;51:797-801.

7. Kolstad A, Holte H, Fosså A, et al. Pneumocystis jirovecii pneumonia in B-cell lymphoma patients treated with the rituximab-CHOEP-14 regimen. Haematologica 2007;92:139-40.

8. McLaughlin P, Grillo-López AJ, Link BK, et al. Rituximab chimeric anti-CD20 monoclonal antibody therapy for relapsed indolent lymphoma: half of patients respond to a four-dose treatment program. J Clin Oncol 1998;16:2825-33.

9. Ennishi D, Terui Y, Yokoyama M, et al. Increased incidence of interstitial pneumonia by CHOP combined with rituximab. Int J Hematol 2008;87:393-7.

10. Katsuya H, Suzumiya J, Sasaki H, et al. Addition of rituximab to cyclophosphamide, doxorubicin, vincristine, and prednisolone therapy has a high risk of developing interstitial pneumonia in patients with non-Hodgkin lymphoma. Leuk Lymphoma 2009;50:1818-23.
11. Lee J, Kim YH, Kang JY, et al. Korean Guidelines for Diagnosis and Management of Interstitial Lung Diseases: Part 3. Idiopathic Nonspecific Interstitial Pneumonia. Tuberc Respir Dis (Seoul) 2019;82:277-84.

12. King TE, Jr. Clinical advances in the diagnosis and therapy of the interstitial lung diseases. Am J Respir Crit Care Med 2005;172:268-79.

13. Chen WL, Tsao Y'T, Chang TH, et al. Impact of interstitial pneumonia on the survival and risk factors analysis of patients with hematological malignancy. Biomed Res Int 2013;2013:185362.

14. Department of Health and Human Services. Panel on Clinical Practices for Treatment of HIV Infection.; Henry J. Kaiser Family Foundation. Panel on Clinical Practices for Treatment of HIV Infection. Guidelines for the use of antiretroviral agents in $\mathrm{HIV}$-infected adults and adolescents, January 28, 2000 by the Panel on Clinical Practices for Treatment of HIV Infection. HIV Clin Trials 2000;1:60-110.

15. Macon WR, Salhany KE. T-cell subset analysis of peripheral $\mathrm{T}$-cell lymphomas by paraffin section immunohistology and correlation of CD4/CD8 results with flow cytometry. Am J Clin Pathol 1998;109:610-7.

16. Azoulay É, Bergeron A, Chevret S, et al. Polymerase chain reaction for diagnosing pneumocystis pneumonia in non-HIV immunocompromised patients with pulmonary infiltrates. Chest 2009;135:655-61.

17. Masur H, Brooks JT, Benson CA, et al. Prevention and treatment of opportunistic infections in HIV-infected adults and adolescents: Updated Guidelines from the Centers for Disease Control and Prevention, National Institutes of Health, and HIV Medicine Association of the Infectious Diseases Society of America. Clin Infect Dis 2014;58:1308-11.

18. Hashimoto K, Kobayashi Y, Asakura Y, et al. Pneumocystis jiroveci pneumonia in relation to CD4+ lymphocyte count in patients with B-cell non-Hodgkin lymphoma treated with chemotherapy. Leuk Lymphoma 2010;51:1816-21.

19. Huang YC, Liu CJ, Liu CY, et al. Low absolute lymphocyte count and addition of rituximab confer high risk for interstitial pneumonia in patients with diffuse large B-cell lymphoma. Ann Hematol 2011;90:1145-51.

20. Koskela HO, Salonen PH, Romppanen J, et al. Longterm mortality after community-acquired pneumonia-impacts of diabetes and newly discovered hyperglycaemia: a prospective, observational cohort study. BMJ Open 2014;4:e005715.

21. Schuetz P, Friedli N, Grolimund E, et al. Effect of 
hyperglycaemia on inflammatory and stress responses and clinical outcome of pneumonia in non-critical-care inpatients: results from an observational cohort study. Diabetologia 2014;57:275-84.

22. Schuetz P, Castro P, Shapiro NI. Diabetes and sepsis: preclinical findings and clinical relevance. Diabetes Care 2011;34:771-8.

23. Delamaire M, Maugendre D, Moreno M, et al. Impaired leucocyte functions in diabetic patients. Diabet Med 1997;14:29-34.

24. Alexiewicz JM, Kumar D, Smogorzewski M, et al. Polymorphonuclear leukocytes in non-insulin-dependent diabetes mellitus: abnormalities in metabolism and

Cite this article as: Li C, Lu F, Lei T, Yu H, Yang H. Clinical features, treatment and risk factors for interstitial pneumonia in B-cell non-Hodgkin lymphoma patients. Transl Cancer Res 2020;9(9):5139-5146. doi: 10.21037/tcr-20-988 function. Ann Intern Med 1995;123:919-24.

25. Liberatore RR Jr, Barbosa SF, Alkimin M, et al. Is immunity in diabetic patients influencing the susceptibility to infections? Immunoglobulins, complement and phagocytic function in children and adolescents with type 1 diabetes mellitus. Pediatr Diabetes 2005;6:206-12.

26. Spatz M, Eibl N, Hink S, et al. Impaired primary immune response in type- 1 diabetes. Functional impairment at the level of APCs and T-cells. Cell Immunol 2003;221:15-26.

27. Edwards MS, Fuselier PA. Enhanced susceptibility of mice with streptozotocin-induced diabetes to type II group B streptococcal infection. Infect Immun 1983;39:580-5. 